\title{
Outcome of Surgical Treatment for Displaced Acetabular Fractures
}

\author{
A Anizar-Faizi, MD UKM, A Hisam, MS Orth, KP Sudhagar*, MS Orth, M Moganadass*, MS Orth, \\ C Suresh*, MS Orth \\ Department of Orthopedics and Traumatology, Universiti Kebangsaan Malaysia Medical Centre, \\ Kuala Lumpur, Malaysia \\ *Department of Orthopedics and Traumatology, Hospital Sultanah Bahiyah, Alor Setar, Malaysia
}

\begin{abstract}
The aim of this study was to assess the outcome of surgical treatment in displaced acetabular fractures in our local facilities. Previous studies reveal good outcome via open reduction method with achievement of congruent joint. We note that studies in this respect have not been conducted in our region. We retrospectively analyzed thirty patients with acetabular fractures who underwent open reduction and internal fixation. The data collection was performed over a 4-year period, from 2008 to 2011. The results showed 20 out of 30 patients (66.7\%) had excellent/good results (Harris Hip Score equal or more than 80 ). Post operative complications were deep infection $(6.7 \%)$, iatrogenic sciatic nerve injury $(10.0 \%)$, avascular necrosis $(16.7 \%)$, heterotopic ossificans (3.3\%), degenerative changes in hip joint $(43.3 \%)$ and loss of reduction $(3.3 \%)$. In conclusion, surgical treatment of displaced acetabular fractures produces good functional outcome despite the complications.
\end{abstract}

\section{Key Words:}

Acetabular fracture, outcome, Harris Hip Score

\section{INTRODUCTION}

The treatment of acetabular fracture is a complex topic. In the past, the non-operative treatment had been practiced as not many surgeons comprehended the normal and pathological anatomy of acetabulum together with concept of surgical approaches for the pelvis. After the classic paper of Judet et al ', in 1964, surgeon's understandings and concepts of treatment have changed. Although it was not popular until 1982, the trend of treatment evolved dramatically. Currently, surgical treatment has evolved to be the treatment of choice as restoration of joint congruity is of paramount importance to reduce the incidence of early hip osteoarthritis ${ }^{2}$.

The outcome of the fixation is dependent on many variables such as energy level of the injury, radiographic fracture pattern, surgeon's knowledge of pelvic anatomy, timing of open reduction and internal fixation (ORIF) and appropriate choice of surgical approach ${ }^{3,45}$. The anatomical and radiographic classification plays an important role and acts as a first step in decision making for the mode of treatment. In addition to this, timing of surgery is crucial as it may influence the outcome. Generally, internal fixation should be performed within the first 10 days of trauma provided the patient is surgically fit ${ }^{6}$. Surgery may be delayed if patient is not fit, but it is advisable to perform the surgery within three weeks as there are difficulties in accurate reduction after this period $^{7,8}$. The outcome after surgery also depends on the experience of the surgeon. It has been demonstrated that specific surgical techniques and experience are critical to achieve satisfactory results.

This study is aimed to concentrate on the outcome of surgical treatment for displaced acetabular fracture in the local setting.

\section{MATERIALS AND METHODS}

This is a retrospective clinical study 30 patients in the Orthopedic Department, Hospital Sultanah Bahiyah, Alor Setar, Kedah, who underwent operative treatment for displaced acetabulum fractures, during the period from January 2008 till December 2011. All thirty patients were followed up for one year duration. Inclusion criteria's in this study were patients with acetabular fractures treated surgically, with fractures involving other limbs, no history of previous injury to the hip or acetabulum and no co-existing hip osteoarthritis. Patients who had defaulted follow up, amd those with previous history of hip injuries and preexisting hip osteoarthritis were excluded. The fractures were classified using Letournal and Judet classification. All patients were assessed and reviewed during follow up in the orthopedic clinic using Harris hip score and radiographic images of pelvis and hip joint. During review the patients' clinical progress were recorded including post-operative

Corresponding Author: Mohd Hisam bin Mohd Ariffin, Department of Orthopedics and Traumatology, Universiti Kebangsaan Malaysia Medical Centre, Kuala Lumpur, Malaysia

Email: hesam8791@yahoo.com 
Table I: Correlation between congruency of fracture and functional score

\begin{tabular}{|lcc|}
\hline Radiograph hip findings & Good/Excellent & Harris Hip Score \\
& Poor/ Fair \\
\hline Congruent & 20 & 2 \\
Non congruent & 1 & 7 \\
\hline
\end{tabular}

complications. Spearman's Rank Correlation Coefficient for non-linear relationship and non-bivariate for normal distribution of data were used as statistical analytical tools.

\section{RESULTS}

The average age of the 30 patients at presentation was 39.9 years old range 14 to 81 years), 23 male and 7 female. Road traffic accident $(86.7 \%)$ was the common cause of injury, followed by alleged fall (13.3\%). The right acetabulum was involved in 17 patients and the left in 13 . Seventeen cases $(56.7 \%)$ were elementary fractures while 13 cases $(43.3 \%)$ were associated type fractures. Commonest pattern of acetabular fractures was posterior wall in 14 patients followed by bicolumnar in six patients (Fig 1).

Three different surgeons were involved in performing the operative procedures.

Average time from the onset of presentation to the surgery was 16.1 days (range 1 day to 68 days). Sixteen patients $(53 \%)$, were operated within two weeks of injury. There was one isolated patient with polytrauma in whom the surgery was performed 68 days after injury. The fixation of acetabulum could not be carried out earlier as the patient was not stable following fixation of other long bones and had been admitted to the Intensive Care Unit. The fixation of the acetabulum was performed - 68 days later as the patient was a young 13 years old boy and had associated subluxation of the right hip joint. Recent follow up of this young boy revealed avascular necrosis of the right femoral head. The surgical approaches used were KocherLangenbach in 18 cases $(60.0 \%)$, illioinguinal in seven $(23.3 \%)$ and combined Kocher-Langenbach with illioinguinal in 5 (16.7\%). In patients who were operated after delay of more than 2 weeks the average duration of surgery approximately 297.7 minutes in comparison to 207.2 minutes in patients who had the surgery after a waiting period of less than 2 weeks.

Post-surgical complications identified were, deep infection in two patients $(6.7 \%)$, iatrogenic sciatic nerve injury in three patients $(10.0 \%)$, avascular necrosis of femoral head in five (16.7\%), heterothropic ossificans in one patient (3.3\%) and degenerative changes of hip joint over a period of time in 16 patients $(43.3 \%)$. Loss of reduction was identified in one patient and was subjected to revision surgery.
Reconstruction plate was the commonest implant used in surgery. Reviewing functional outcome, 13 patients of elementary acetabular fractures and seven patients of the associated type of acetabular fractures had Harris Hip Score equal or more than 80 (considered as good/excellent). Remaining four patients with elementary acetabular fractures and six patients with associated type fractures had poor/fair (less than 80) Score.

Correlating restoration of joint congruency following a fracture fixation with functional score, 20 out of 30 patients with congruent joint radiography scored good/excellent Harris Hip Score. Seven patients with non-congruent joint radiography, had poor/fair score (Table 1). Congruent reduction was defined as achievement of anatomical reduction in the operated acetablum and hip compared with antero-posterior radiograph of the contralateral normal joint. Patients with poor restoration of the five radiographic pelvic lines (ilio-inguinal, ilio-pectineal, dome, posterior wall and anterior wall), inward subluxation of the hip joint and loss of parallelism were included in the incongruent group.

The duration of waiting period for surgery following the injury showed a correlation with functional outcome using Harris Hip Score. There was a significant nonparametric correlation between duration of waiting period for surgery (days) and Harris Hip Score with p-value $=0.007$. However the observed correlation coefficient (r) is 0.487 , suggests negative and moderate correlation. In conclusion, shorter duration of waiting period for surgery gave higher Harris Hip Score (Figure 5).

There was no significant correlation between age of patient and Harris Hip Score (p-value=0652). Since the correlation coefficient (r) is 0.086 , near to zero, hence no correlation exists between age and Harris Hip score (Figure 6).

\section{DISCUSSION}

The management of displaced acetabular fractures remains a challenging task for orthopaedic surgeons. This statement is still applicable in some countries but is being progressively eliminated with availability and dissemination of knowledge via information technology and online international literatures. This has helped in setting up the standardization of clinico-radiological assessment, classification of fractures, surgical approaches and fixation techniques of the fractures. 


\section{Pattern of fractures (\%)}

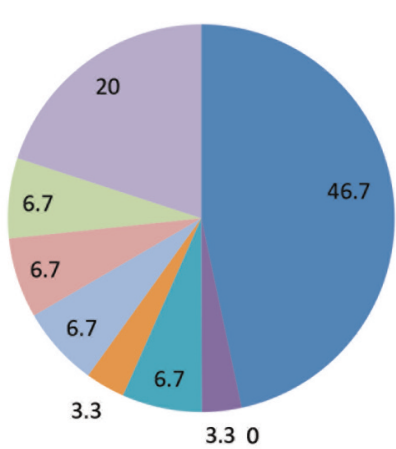

" post wall

vost column

II ant wall

ant column

transverse

T-shape

post wall and column

"transverse with post wall

post hemitransverse

m both column

Fig. 1: Pattern of acetabular fracture in percentage. Post-posterior, ant- anterior.
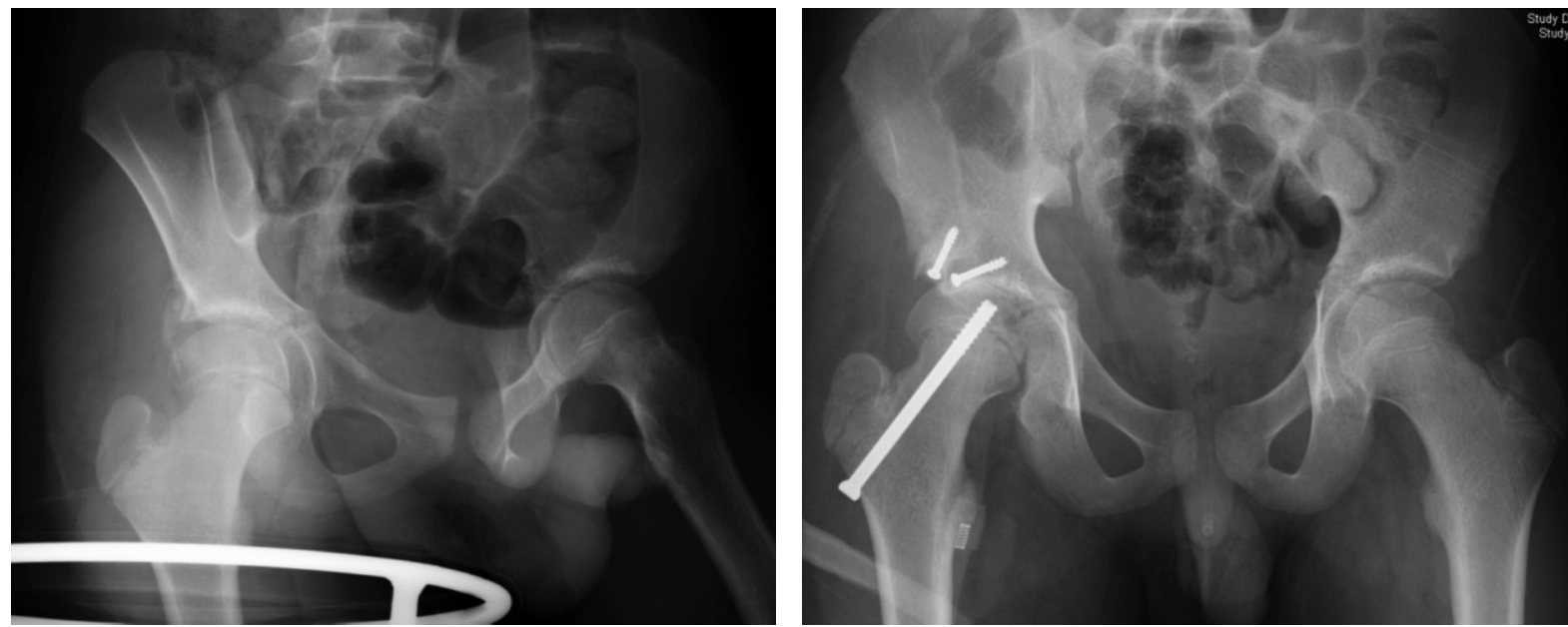

Fig. 2: Pre op (right) and postop (left) radiograph of 13 years old boy who was operated 68 days after polytrauma injury.
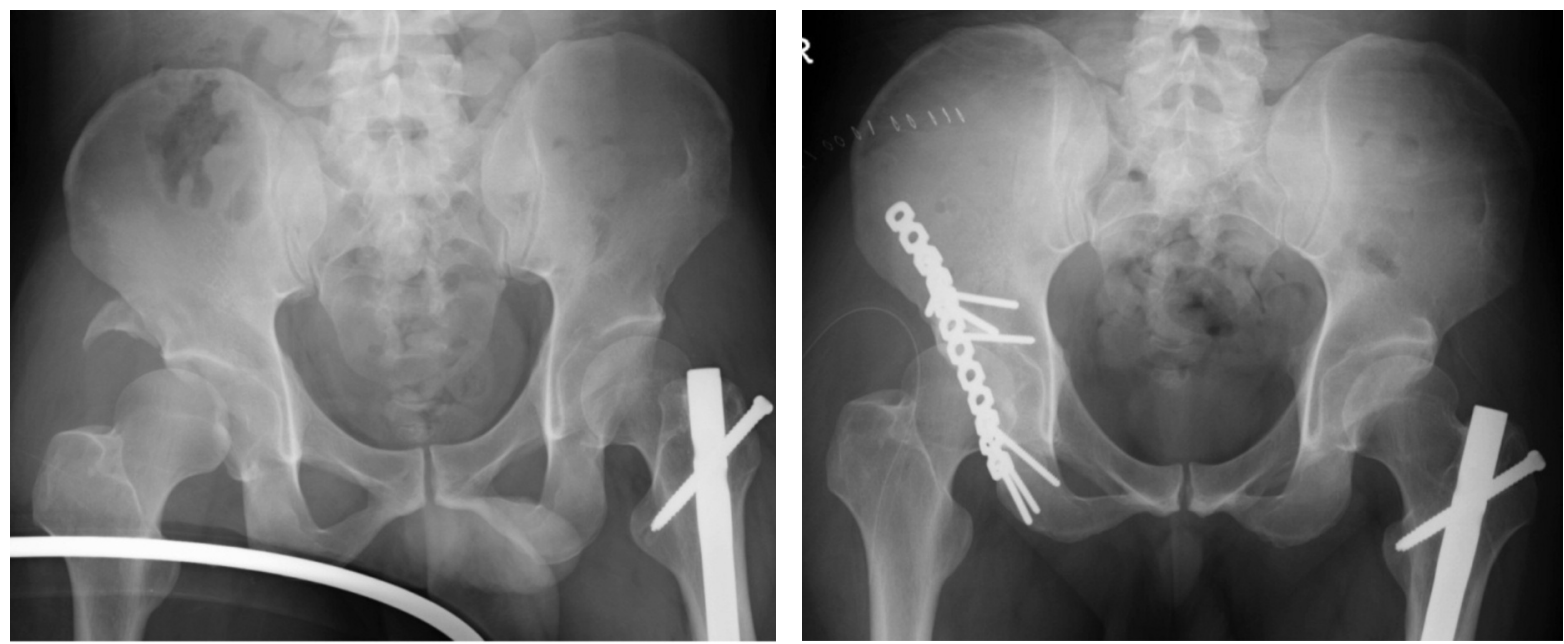

Fig. 3: Pre-op (left) and post-op (right) radiograph of the patient with good/excellent result. 

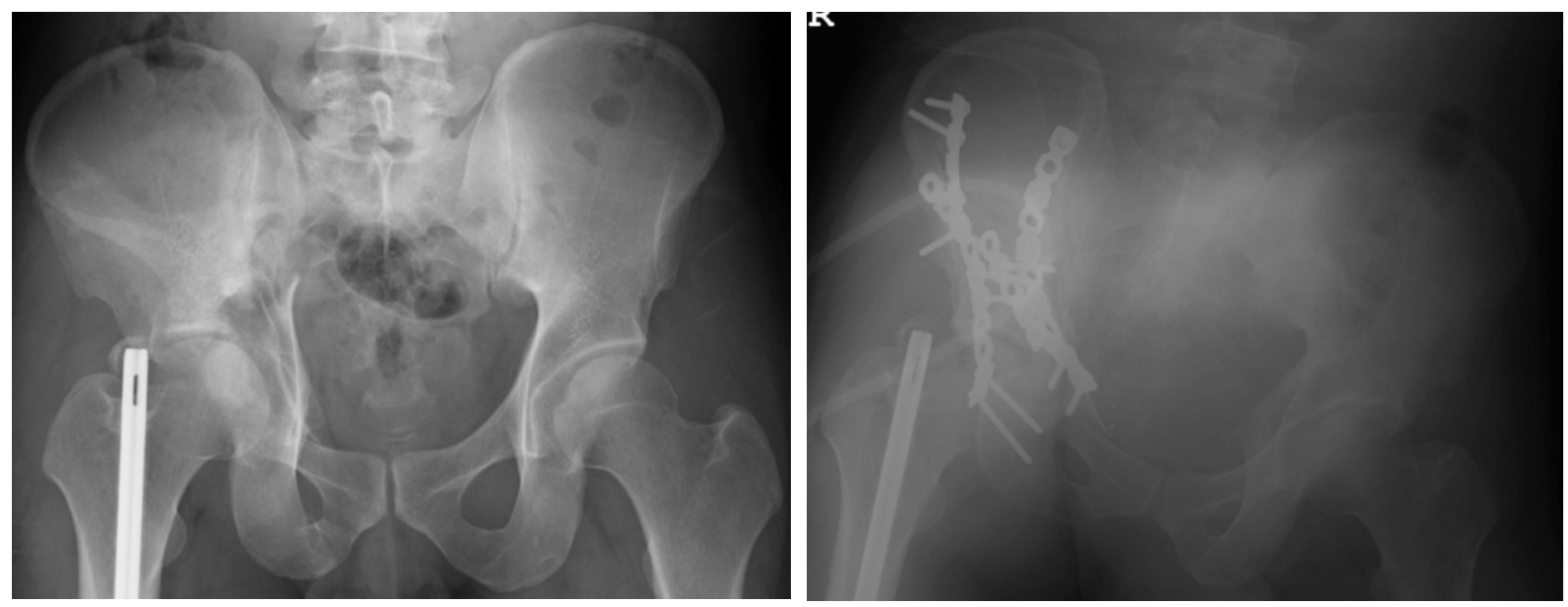

Fig. 4: Pre-op (right) and post-op (left) radiograph in-patient with poor/fair result.

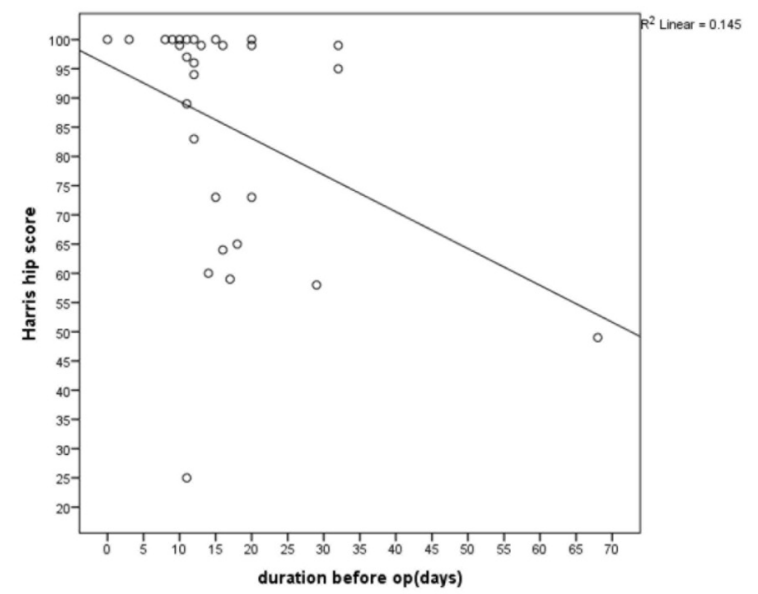

Fig. 5: Correlation of waiting period for surgery (days) with Harris Hip Score.

The Kocher-Langenbach approach is the commonest surgical approach used and it was the most suitable for the fracture patterns presenting in our centre:. 14 cases with posterior wall fracture, both columns in six, two each of transverse, posterior wall and column, transverse with posterior wall and posterior hemitransverse; and one each of T-shape and anterior column. The other approaches (Illioinguinal and combined) was used depending on the other types and fracture patterns. However, the operative time taken was longer in the illioinguinal approach (average $264.2 \mathrm{~min}$ ) compared to Kocher-Langenbach approach (average $199.0 \mathrm{~min}$ ), probably due to greater familiarity with the latter approach.

As various previous studies had revealed, the congruency of the fracture site correlated directly with the functional outcome of displaced acetabular fractures ${ }^{9,10,11}$. Open

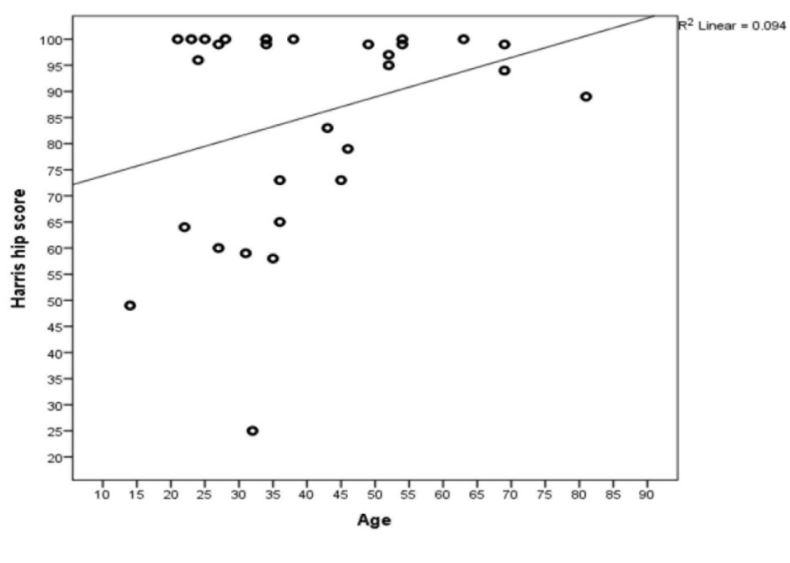

Fig. 6: Correlation of age and Harris Hip Score.

reduction remains the best method to achieve good anatomical reduction, as borne out in our study. (Table I). Twenty patients $(66.7 \%)$ who had achieved radiographic congruency had good/excellent Harris Hip Score, while seven patients $(23.3 \%)$ who did not achieve radiographic congruency had poor/fair Harris Hip Score. Although congruity of fractures had been achieved, there were two patients who had poor/fair functional outcome due to deepseated infection which had affected their rehabilitation program leading to poor functional score.

Timing for surgery has been shown to be very important as several studies reported poor results when open reduction and internal fixation was performed more than three weeks post injury. Johnson et al (1994) $)^{12}$ published their study regarding poor clinical results in delayed reconstruction of acetabular fractures of more than 21 days compared to early 
intervention. Our study also showed similar findings as patients who had undergone operation earlier scored higher Harris Hip Score.

In the elderly population, gaining sound and secure fixation in osteoporotic pelvic bone can be unpredictable. Helfet et $a l^{13}$, reported good to excellent outcomes at three years in 18 patients with an average age 67 years. But several of the patients had significant risk factors suggesting potential need for future total hip replacement. Their study also outlined the importance of determining appropriate surgical indication among elderly patients, such as fracture pattern amenable to fixation through a single non-extensile exposure without performing a trochanteric osteotomy or disrupting the abductor musculature, adequate bone quality for fixation, no femoral head injury, and a reasonable surgical time (3-4 hours). In our study, there was no correlation between age and Harris Hip Score which would mean that elderly patients who had undergone surgery treatment could still have good functional outcome. However, the patient selection in the elderly is crucial in order to obtain good outcome. For those whose general health condition contraindicates surgery, conservative management is the treatment of choice ${ }^{14}$.

The infection rate in our study was $6.7 \%$ (2 patients), slightly higher compared to others studies ${ }^{15,16}$. Both these patients developed deep-seated infection. Wound debridement was performed and treated with two weeks course of intravenous antibiotics. However, due to delay in rehabilitation, the functional score in both these patients was poor/fair. There was one patient who had heterotopic ossificans and the main contributing factor was the amount of subperiosteal stripping. Other factors which contribute to heterotopic ossificans are severity of the fracture pattern and the use of osteotomies during surgery ${ }^{17}$. Surprisingly, this condition did not really affect the functional score as reported by Oransky et $a{ }^{18}$. We routinely use prophylactic NSAIDs as there was demonstrated efficacy of reduction in heterotopic ossificans formation in post operative patients ${ }^{19}$.
The incidence of avascular necrosis in various studies varies from $3 \%$ to $53 \% 2$. The value was quite high in our centre (5 out of 30 or $16.7 \%$ ). - The surgical approach which included opening the capsule may be the main cause of avascular necrosis together with early learning curve of the surgeon. Three patients had developed sciatic nerve injury, of whom only one - had full recovery at 10 months post-operative, the others had permanent injury.

Limitation in this study was the small number of patients. This was probably due to rarity of the cases compared to other trauma condition. A larger number of patients would have helped us to identify the specific types of fracture patterns with predictable poor prognosis. Long term follow up is emphasized for early detection of poor outcome in the younger patients.

\section{CONCLUSION}

The operative treatment for displaced acetabular fracture gives a satisfactory functional outcome despite its wellknown complications. In our study, the functional outcome is good/excellent in 70 percent of the patients with improved quality of life. Careful planning of the surgery is essential in order to proper assessment of fracture pattern and plan and ensure correct surgical approach. In addition it is important that the surgeon explains the risks of surgery and possible outcomes to the patients particularly when the waiting period for surgery is more than two weeks. 


\section{REFERENCES}

1. Judet R, Judet J, Letournel E. Fractures of the acetabulum: classification and surgical approaches for open reduction: preliminary report. J Bone Joint Surg Am 1964; 46-:1615-46.

2. Matta JM, Anderson LM, Epstein HC. Fractures of the acetabulum: a retrospective analysis. Clin Orthop 1986; 205: 230-40.

3. Mast J, Jakob R, Ganz R. Planning and reduction technique in fracture surgery. Springer, Berlin Heidelberg 1989: 54-6.

4. Mears DC, Velyvis JH, Chang C-P. Displaced acetabular fractures managed operatively: indicators of outcome. Clin Orthop 2003; 407: 173-86.

5. Stannard JP, Alonso JE. Controversies in acetabular fractures. Clin Orthop 1998; 353: 74-80.

6. Letournel E. Acetabulum fractures: classification and management. Clin Orthop 1980; 151: 81-106.

7. Brueton RN. A review of 40 acetabular fractures: the importance of early surgery. Injury 1993; 24: 171-4.

8. Kebaish AS, Roy A, Rennie W. Displaced acetabular fractures:long-term follow-up. J Trauma 1991; 31: 1539-42.

9. Mears DC, Velyvis JH, Chang CP. Displaced acetabular fractures managed operatively: indicators of outcome. Clin Orthop Relat Res 2003; 407: 173-86.

10. Estrems-Díaz V, Hernández-Ferrando L \& Balaguer-Andrés J. Acetabular fractures: Short term results. Rev. Esp Cir Ortop Traumatol 2012; 56(1): 17-23.

11. Dunet B, Tournier C \& Billaud A. Acetabular fracture: Long-term follow-up and factors associated with secondary implantation of total hip arthroplasty. Orthop \& Traumatol Surg Res 2013; 99: 281-90.

12. Johnson EE, Matta JM, Mast JW, Letournel E. Delayed reconstruction of acetabular fractures 21-120 days following injury. Clin Orthop Relat Res 1994; 305: 20-30.

13. Helfet DL, Borrelli J, DiPasquale T. Stabilization of acetabular fractures in elderly patients. J Bone Joint Surg Am1991; 74-75365.

14. Guerado E, Canoa JR, Cruza E. Fractures of the acetabulum in elderly patients: An update. Injury 2012; 43(2): $33-41$.

15. Liebergall M, Mosheiff R \& Low J. Acetabular fractures. Clinical outcome of surgical treatment. Clin Orthop Relat Res 1999; 366: 205-16.

16. Giannoudis PV, Grotz MR \& Papakostidis C. Operative treatment of displaced fractures of the acetabulum, a meta-analysis. $J$ Bone Joint Surg Br 2005; 87-24-9.

17. Kaempffe FA, Bone LB, Border JR. Open reduction and internal fixation of acetabular fractures: heterotopic ossification and other complications of treatment. J Orthop Trauma 1991; 5(4): 439-45.

18. Oransky M, Sanguinetti C. Surgical treatment of displaced acetabular fractures: results of 50 consecutive cases. J Orthop Trauma 1993; 7(1): 28-32.

19. Burd TA, Lowry KJ, Anglen JO. Indomethacin compared with localized irradiation for the prevention of heterotopic ossification following surgical treatment of acetabular fractures. J Bone Joint Surg Am 2001; 83: 1783-8. 